APLICAÇÃO DA TÉCNICA DE ELETROFLOCULAÇÃO UTILIZANDO CORRENTE ALTERNADA NO TRATAMENTO DE ÁGUA DE PRODUÇÃO DA INDÚSTRIA DO PETRÓLEO

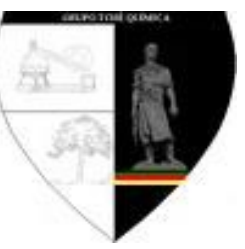

\title{
APPLICATION OF THE TECHNIQUE OF ELECTROFLOCCULATION USING ALTERNATE CURRENT IN TREATMENT OF WATER PRODUCTION FROM OIL INDUSTRY
}

\author{
CERQUEIRA, Alexandre Andrade1; MARQUES, Mônica Regina da Costa²; RUSSO, Carlos ${ }^{3}$
}

1,2,3 Universidade do Estado do Rio de Janeiro, Instituto de Química, Laboratório de Tecnologia Ambiental, LABTAM, Rua São Francisco Xavier, 524 PHLC sala 304, Cep 20550-013 - Maracanã - RJ - Brasil

* e-mail: alexandrecerq@ig.com.br

Received 4 November 2009; received in revised form 7 January 2010; accepted 9 January 2010

\section{RESUMO}

O presente trabalho visa à realização do tratamento de água de produção da indústria do petróleo, o qual é o principal resíduo gerado durante o processo de exploração e produção. Esta água é composta, principalmente, por produtos químicos tóxicos, sais inorgânicos e metais pesados, os quais dificultam seu tratamento e disposição final. Apesar de, ultimamente, esta água de produção ser reinjetada em poços de produção para aumentar a pressão dos mesmos, ela ainda não apresenta a qualidade desejada para tal, daí decorrendo um problema a ser resolvido. Para o tratamento desse efluente várias tecnologias estão sendo estudadas, sendo que o objetivo deste trabalho é o de estudar a exeqüibilidade da aplicação do processo de eletrofloculação utilizando corrente alternada de frequência variável no tratamento desse efluente simulado. Os equipamentos de eletrofloculação utilizados foram de corrente contínua e corrente alternada de frequência variável e como material dos eletrodos utilizou-se o alumínio, sendo todas as etapas realizadas em sistema de batelada. As análises relacionadas à caracterização do efluente bruto e o eletrofloculado foram os seguintes: óleos e graxas, turbidez, cor, $\mathrm{pH}$ e condutividade. Os resultados mostraram que foi possível obter remoções de óleos e graxas, cor e turbidez em valores acima de $98 \%$.

Palavras-chave: Eletrofloculação, Água de Produção, Tratamento de Efluentes, Produção de Petróleo

\begin{abstract}
This work is related to the electroflocculation process applied to the treatment of the production water, which is the main waste generated during the process of exploration and production of oil. This wastewater is contains toxic chemicals, inorganic salts and heavy metals, which hinders its treatment and final disposal. Lately, this water is being re-injected into the production wells to increase the pressure, but its quality is not adequate. For the treatment of this effluent various technologies have been proposed, and the aim of this paper is to study the feasibility of application of the electroflocculation using alternate current variable frequency in the treatment of a simulated wastewater. The equipment used both direct and variable frequency alternate current. Aluminium was the material as the electrodes, and all runs were carried in a batch system. The analysis related to the characterization of the raw and electroflocculated wastewater were: oil and grease, turbidity, color, $\mathrm{pH}$ and conductivity. The results showed that it was possible to obtain removal efficiencies of oils and greases, color and turbidity above $98 \%$.
\end{abstract}

Keywords: Electroflocculation, Water Production, Wastewater Treatment, Oil Production 


\section{Introdução}

A poluição das águas por matéria oleosa constitui um grande problema ambiental, uma vez que a indústria do petróleo produz este efluente em todas as suas etapas de exploração, produção, transporte e refino (Mariano, 2005). A indústria de extração de petróleo, ciente da crescente preocupação com os problemas ambientais que possam ser causados através da toxicidade de seus efluentes, bem como do surgimento de novas leis e resoluções ambientais mais restritivas, procura enquadrar-se dentro destas novas exigências. Um dos pontos cruciais a serem atacados é a questão da água de produção, que é um subproduto da extração do petróleo, a qual vem aumentando gradativamente seu volume à medida que os poços vão envelhecendo e que novos poços são perfurados (Campos et al, 2005).

Durante a vida econômica de um poço de petróleo, a geração de água de produção pode chegar a exceder em dez vezes o volume de produção do óleo (Vieira, 2003), pois constituem um efluente complexo, de salinidade elevada, cuja composição pode variar dependendo do tipo de campo e de sua idade, da sua origem e da qualidade do óleo (Rodrigues, 2007).

O descarte de efluentes de água de produção deve ser tratado de acordo com a legislação ambiental. $\mathrm{Na}$ extração de petróleo, existe uma regulamentação específica relacionada à água de produção, bem como da água de re-injeção. A Resolução impõe o tratamento de efluentes na extração em plataformas marítimas e conceitua a água de produção no Art $2^{\circ}$, I como "a água normalmente produzida junto com o petróleo" (CONAMA $n^{\circ}$ 393/07), também denominada água de processo ou produzida.

Atualmente, a utilização dos derivados de petróleo está tão inserida no dia-dia que sua extinção agora, não só pelos automóveis que não trafegariam, mas também pela utilização de bens de consumo como canetas, camisas, pen drives, computadores etc., se tornaria inviável. Isto demonstra o quão a sociedade é dependente desta matéria-prima. Assim, a avaliação da eletrofloculação com corrente alternada no tratamento de efluentes proveniente de plataformas off-shore possui grande relevância, para que seja desenvolvido um processo de tratamento que seja rápido, eficiente e de custo viável.

Nos últimos anos, o processo de eletrofloculação vem crescendo muito, principalmente no que diz respeito à sua simplicidade de operação e aplicação em diversos tipos de efluentes.

Atualmente, várias tecnologias vêm sendo desenvolvidas visando a redução significativa de óleos e graxas (O\&G) da água de produção. Entre elas pode-se citar filtração em leito absorvente, adsorção em carvão ativo, biooxidação entre outros, porém, estas tecnologias em geral, ainda não são viáveis tanto do ponto de vista técnico quanto econômico para o tratamento da água em plataforma, devido às limitações de espaço físico e peso desses equipamentos, dificuldade de execução de obras no ambiente marítimo e também tempo de residência destas águas em plataformas, que, em geral, não excede de 15 minutos (Gabardo, 2007).

\subsection{Eletrofloculação (EF)}

A eletrofloculação é um processo que envolve a geração de coagulantes "in situ" pela dissolução de agentes floculadores in situ a partir de eletrodos de ferro e alumínio, pela ação da corrente elétrica aplicada a esses eletrodos. A geração de íons metálicos ocorre no anodo, enquanto o gás hidrogênio surge no catodo (Figura 1).

\subsection{Tipos de reações envolvidas no processo de eletrofloculação}

Segundo Mollah et al (2001), o mecanismo da eletrofloculação é altamente dependente da química do meio aquoso, especialmente a condutividade. Além disso, outras características, tais como, $\mathrm{pH}$, tamanho da partícula e concentração dos constituintes, influenciam também 0 processo de eletrofloculação.

As reações do alumínio estão descritas a seguir.

\subsubsection{Reações do alumínio}

Oxidação do Al sólido (reação anódica)

$\mathrm{Al} \rightarrow \mathrm{Al}^{3+}{ }_{(\mathrm{aq})}+3 \mathrm{e}^{-}$

Solvatação do cátion formado

$\mathrm{Al}^{3+}{ }_{(\mathrm{aq})}+6 \mathrm{H}_{2} \mathrm{O} \rightarrow \mathrm{Al}\left(\mathrm{H}_{2} \mathrm{O}\right)_{6}{ }^{3+}$

Formação do agente coagulante

$\mathrm{Al}\left(\mathrm{H}_{2} \mathrm{O}\right)_{6}{ }^{3+} \rightarrow \mathrm{Al}(\mathrm{OH})_{3}(\mathrm{~s})+3 \mathrm{H}^{+}+3 \mathrm{H}_{2} \mathrm{O}$

Reações secundárias

${ }_{n} \mathrm{Al}(\mathrm{OH})_{3} \rightarrow \mathrm{Al}_{\mathrm{n}}(\mathrm{OH})_{3 \mathrm{n}}(\mathrm{s})$ 
Entretanto, dependendo do $\mathrm{pH}$ do meio aquoso, outras espécies iônicas como $\mathrm{Al}(\mathrm{OH})^{2+}$, $\mathrm{Al}_{2}(\mathrm{OH})_{2}{ }^{4+}$ e $\mathrm{Al}(\mathrm{OH})_{4}{ }^{-}$também podem estar presentes no sistema (Mollah et al., 2001).

Pode-se notar que, pela Reação 4, vários complexos de alumínio podem ser formados em meio aquoso, os quais conferem uma característica gelatinosa ao meio (Crespilho \& Rezende, 2004).

Com o aumento do $\mathrm{pH}$, as diferentes formas de hidróxidos de alumínio, formadas durante o processo eletrolítico, tendem a ficar solúveis (Chen et al, 2000), devido às reações de complexação com íons $\mathrm{OH}^{-}$. Assim, uma parte do alumínio gerado e solúvel não participa das reações de coagulação e tende a ficar em solução, principalmente na forma de $\mathrm{Al}(\mathrm{OH})_{4}$. $\mathrm{Na}$ hidrolise do $\mathrm{Al}^{3+}$, quando $\mathrm{oH}>7,0$, a formação dos complexos de hidróxidos de alumínio solúveis é favorecida (Crespilho \& Rezende, 2004).

De acordo com Kim et al (2002) no caso de anodos $\mathrm{Al}$, o principal mecanismo de coagulação a pH na faixa de 2 a 3 é por compressão da dupla camada. Quando o pH inicial é de 4 a 9 , a coagulação é realizada por adsorção, ou neutralização das cargas.

As espécies químicas dominantes são também diferentes de acordo com o pH inicial; $\mathrm{Al}^{3+}$ e Al $(\mathrm{OH})_{2}{ }^{+}$são predominantes em $\mathrm{pH} 2$ a 3 , e quando $\circ \mathrm{pH}$ é em 4 a 9 espécies poliméricas tais como $\mathrm{Al}_{13} \mathrm{O}_{4}(\mathrm{OH})_{24}{ }^{7+}$, são formadas $\mathrm{e}$ precipitadas como $\mathrm{Al}(\mathrm{OH})_{3}(\mathrm{~s})$. Quando o valor de $\mathrm{pH}$ é superior a 10, a presença de ânions monoméricos, $\mathrm{Al}(\mathrm{OH})_{4}^{-}$é aumentada, enquanto que a de $\mathrm{Al}(\mathrm{OH})_{3}(\mathrm{~s})$ é diminuída.

As complexidades inerentes aos processos de eletrofloculação, e também a presença de processos secundários, como por exemplo a hidrólise da água (equação 5), tornam bastante complexa sua natureza (Kobya et al, 2003).

$$
3 \mathrm{H}_{2} \mathrm{O}+3 \mathrm{e} \rightarrow 3 / 2 \mathrm{H}_{2}+3 \mathrm{OH}^{-} \text {(catodo) }
$$

Entretanto, se o potencial no anodo é suficientemente alto, também podem ocorrer reações secundárias, tais como, oxidação de compostos orgânicos pelos íons $\mathrm{Cl}^{-}$presentes nos efluentes (Vlyssides et al, 1999).

$2 \mathrm{Cl}^{-} \rightarrow \mathrm{Cl}_{2}+2 \mathrm{e}$
$\mathrm{Al}^{3+}{ }_{(a q)}$ e íons $\mathrm{OH}^{-}$gerados pelo eletrodo nas reações (1) e (5) reagem de modo a formar várias espécies monoméricas: espécies como o $\mathrm{Al}(\mathrm{OH})^{2+}, \quad \mathrm{Al}(\mathrm{OH})_{2}{ }^{+}, \quad \mathrm{Al}_{2}(\mathrm{OH})_{2}{ }^{4+}, \quad \mathrm{Al}(\mathrm{OH})_{4}{ }^{-}, \quad$ e espécies poliméricas como: $\mathrm{Al}_{6}(\mathrm{OH})_{15^{3+}}$, $\mathrm{Al}_{7}(\mathrm{OH})_{17^{4+}}, \quad \mathrm{Al}_{8}(\mathrm{OH})_{20}{ }^{4+}, \quad \mathrm{Al}_{13} \mathrm{O}_{4}(\mathrm{OH})_{24}{ }^{7+}$, $\mathrm{Al}_{13}(\mathrm{OH})_{34}{ }^{5+}$, que se transformam em $\mathrm{Al}(\mathrm{OH})_{3}$, de acordo com a complexa precipitação cinética das reações (Gurses et al, 2002; Can et al, 2006; Kobya et al, 2003; Bayramoglu et al, 2004).

A formação de diferentes espécies monoméricas e poliméricas também desempenha um importante papel no processo de eletrofloculação. Diversos mecanismos de interações são possíveis entre as moléculas do efluente, a hidrólise depende do $\mathrm{pH}$ do meio e dos tipos de íons presentes. Dois dos principais mecanismos de interação estão sendo considerados como: precipitação e adsorção, cada uma delas proposta para uma faixa distinta de $\mathrm{pH}$. Precipitação ocorre em $\mathrm{pH}$ baixo, enquanto que em $\mathrm{pH}$ superior $(>6,5)$ ocorre adsorção (Can et al, 2006; Kobya et al, 2003; Gurses et al, 2002).

Segundo Mollah et al (2004), estes compostos hidróxidos, poli-hidróxidos e polihidroximetálicos possuem forte afinidade pelas partículas dispersas, bem como os íons que causam a coagulação. Os gases que são formados nos eletrodos são responsáveis pela flotação do material coagulado. A eletrofloculação é um processo que está intrinsecamente associada com a eletroflotação de vez que bolhas de hidrogênio e oxigênio são produzidas no ânodo e cátodo, respectivamente. O êxito do processo de eletrofloculação é determinado pelo tamanho das bolhas, bem como pelo contato das bolhas com o efluente. Acredita-se que as pequenas bolhas proporcionam maior área de contato entre a superfície das partículas no efluente, resultando em uma melhor eficiência do processo.

Uma das maiores vantagens da EF é a remoção de O\&G. Isso ocorre devido à facilidade de coagulação e flotação desses compostos (Silva, 2002). Como conseqüências da interação dessas moléculas com o hidróxido de alumínio, formam-se partículas neutras, cuja densidade é bem menor do que a da água, as quais são carreadas para a superfície do líquido. Além disso, a EF requer equipamentos simples e de 
fácil operação, em que potencial aplicado e a corrente podem ser medidos e controlados de maneira automatizada;

Devido a estas vantagens, o processo de EF tem sido muito investigado, visando à otimização do processo.

Nos últimos anos, o interesse em torno da eletrofloculação vem crescendo muito, principalmente no que diz respeito à sua simplicidade de operação e aplicação em diversos tipos de efluentes. Entretanto o número reduzido de estações de tratamento em funcionamento, especialmente no Brasil, torna-se um obstáculo à sua disseminação.

\subsection{Tecnologia de eletrofloculação com corrente alternada de frequência variável}

Os reatores eletrolíticos atualmente disponíveis funcionam com uma fonte elétrica de corrente contínua. Neste trabalho, foi utilizada a tecnologia de eletrofloculação utilizando corrente alternada, que consiste na alteração da onda eletromagnética de corrente alternada de 60 Hertz comercializada pelas concessionárias de energia, em uma onda com tensão variável e freqüencia maior que $4 \mathrm{~Hz}$ até $40 \mathrm{Hertz}$.

A tecnologia de conversão utilizada simula a formação de corrente contínua, porém com características de alternada. Esta corrente alternada é gerada reconstituindo-se a forma de senóide da corrente elétrica de entrada, em um sistema de conversão com controle vetorial, onde será gerado um pulso controlado com o tempo de formação (período) ajustado por uma base de tempo programável através de um sistema de microprocessadores. Este sistema induz o sistema de disparo de um oscilador a formar uma nova forma de onda e que tem um tempo de permanência de crista grande o suficiente para haver condução em uma dada polaridade.

O objetivo deste trabalho é avaliar a utilização da técnica de eletrofloculação com corrente alternada de frequência variável em relação ao sistema convencional de corrente contínua para o tratamento de efluentes de águas de produção da indústria do petróleo.

\section{Parte Experimental}

\subsection{Procedimentos}

Neste capítulo, encontram-se descritas as etapas de desenvolvimento experimental.

\subsubsection{Unidade de bancada de corrente contínua}

A unidade de bancada é composta por uma fonte de corrente contínua, um transformador isolador que tem a finalidade de separar a rede elétrica alimentadora primária da etapa de retificação/conversão secundária, um indicador de leitura de corrente, um indicador de leitura de tensão. Estes dois últimos destinam-se a orientar o operador quanto aos parâmetros aplicados à pequena colméia de eletrodos.

A Figura 2 consiste em uma célula eletrolítica de vidro com capacidade de $1 \mathrm{~L}$ sob agitação magnética, na qual é inserido verticalmente um eletrodo (monopolar) do tipo colméia, construído com 7 placas de alumínio intercaladas. Estas placas possuem dimensões de $10 \mathrm{~cm}$ de altura e $5 \mathrm{~cm}$ de largura (com espessura de $3 \mathrm{~mm}$ ) e separadas por espaçadores de 0,5 cm de espessura cada.

\subsection{Unidade de bancada de corrente alternada}

A corrente alternada de tensão inferior a $15 \mathrm{~V}$ e frequência variável entre 4 e $40 \mathrm{~Hz}$, foram obtidas a partir de um conversor CA/CA onde o potencial de entrada em $60 \mathrm{~Hz}$ é retificado, e novamente oscilado para se obter um potencial também em corrente alternada, porém, nos níveis desejados.

A Figura 3 apresenta o diagrama do equipamento de CA onde, temos inicialmente um transformador abaixador que reduz a tensão de entrada em corrente alternada de $60 \mathrm{~Hz}$, adequando aos níveis de tensão necessários para a retificação.

$\mathrm{Na}$ etapa de retificação, o potencial é transformado em corrente contínua, em seguida a nova corrente em CC, é tratada no módulo oscilador que converte em pulsos com largura controlada e que formarão a nova forma de onda (CA), com período ajustado por nível de tensão que poderá variar entre 4 e $40 \mathrm{~Hz}$ dependendo do nível de realimentação (referência) proveniente do controlador de carga. 
Desta forma, tem-se uma forma de onda semi-senoidal onde o período varia em função da carga buscando assim, obter-se o melhor rendimento em níveis de potência ativa.

A Figura 4 consiste em uma célula eletrolítica contendo um reator de vidro com capacidade de $1 \mathrm{~L}$, na qual é inserido verticalmente o eletrodo do tipo colméia, construído com sete placas de alumínio intercaladas de $10 \mathrm{~cm}$ de altura, $5 \mathrm{~cm}$ de largura e $1,5 \mathrm{~mm}$ de espessura cada. As placas foram separadas por espaçadores (com $0,5 \mathrm{~cm}$ de espessura cada), o que permitia variar-se a distância entre os eletrodos.

\subsection{Preparo do efluente sintético contendo óleo cru}

Para o preparo da emulsão óleo em água $(\mathrm{O} / \mathrm{A})$, foram utilizadas duas soluções salinas distintas (sintética e natural - água do mar).

A água do mar foi recolhida em Praia Seca (Região dos Lagos, RJ).

Assim que recebido, o óleo cru utilizado neste trabalho foi previamente acidificado com $\mathrm{H}_{2} \mathrm{SO}_{4}$ concentrado a $\mathrm{pH} \leq 2$ e preservado em refrigeração a $\mathrm{T} \leq 4{ }^{\circ} \mathrm{C}$.

\section{Simulação do efluente contendo emulsão (O/A).}

a) Emulsão O/A a partir da solução salina sintética:

$900 \mathrm{~mL}$ de água destilada salinizada com $\mathrm{NaCl}$ (35.000 mg/L) foram transferidos a um becher $(1000 \mathrm{~mL})$ contendo $0,9 \mathrm{~g}$ de óleo cru e $200 \mathrm{mg} / \mathrm{L}$ dos emulsificantes (50\% SP 60 e $50 \%$ TW 60) A emulsão O/A foi então obtida após agitação mecânica vigorosa de aproximadamente 10.000 rpm por 20 minutos. A caracterização da emulsão foi feita por medida de condutividade, $\mathrm{pH}$, turbidez, cor e TOG.

b) Emulsão O/A a partir da água do mar $900 \mathrm{~mL}$ de água do mar foram transferidos a um becher $(1000 \mathrm{~mL})$ contendo $0,9 \mathrm{~g}$ de óleo cru e $200 \mathrm{mg} / \mathrm{L}$ dos emulsificantes (50\% SP 60 e $50 \%$ TW 60). A emulsão O/A foi então obtida após agitação mecânica vigorosa de aproximadamente de 10.000 rpm por 20 minutos. A caracterização da emulsão foi feita por medida de condutividade, $\mathrm{pH}$, turbidez, cor e TOG.

\subsection{Ensaios de Eletrofloculação}

O procedimento experimental consistiu de ensaios de eletrofloculação em corrente alternada e corrente contínua realizados em reatores de batelada utilizando eletrodos de alumínio em células eletrolíticas.

Após adição do efluente na célula eletrolítica, o sistema foi mantido sob agitação constante por meio de agitador magnético.

Depois de transcorrido o intervalo de tempo pré-determinado de eletrólise, aguardavase 30 minutos para que ocorresse a flotação completa do efluente eletrofloculado, e dessa forma, através de um dispositivo na parte inferior, o efluente tratado era transferido para um outro recipiente onde eram retiradas as amostras (alíquotas) para análise.

\subsubsection{Parâmetros analisados}

Amostras dos efluentes bruto $e$ eletrofloculado foram analisadas para se avaliar a eficiência do processo. Os parâmetros analisados foram: condutividade, $\mathrm{pH}$, turbidez, cor e óleos e graxas.

\section{Resultados e Conclusões}

Para a realização deste estudo, foram utilizados efluentes reais de água de produção, efluentes simulados oleosos em água salina sintética e na água do mar. Foi utilizada uma solução salina a $35.000 \mathrm{mg} / \mathrm{L}$, pois, a mesma é a media de salinidade de água do mar (Fonseca, 2001).

A Tabela 1 apresenta os resultados de caracterização dos três efluentes em diferentes meios aquosos (água de produção, água salina sintética e água do mar).

As diferenças observadas nas medidas de condutividade, $\mathrm{pH}$, cor e turbidez na água de produção, no efluente simulado na água salina e no efluente simulado na água do mar, podem ser creditadas aos tipos de sais dissolvidos e suas concentrações encontrados na água do mar e na água de produção.

\subsection{Resultados preliminares}

Estes testes, de caráter meramente exploratório, foram realizados com o objetivo de se conhecer um pouco o efluente de forma a dar início à investigação sistemática dos efeitos estudados. Inicialmente, foram realizados 
experimentos com a finalidade de se determinar o melhor material do eletrodo e verificar o efeito da intensidade de corrente aplicada (A) e tempo de eletrólise na remoção de carga orgânica do efluente em estudo.

\subsection{Seleção do material do eletrodo}

A montagem do eletrodo é o elemento central do tratamento. Assim, a seleção adequada dos seus materiais é muito importante. Os materiais mais comuns do eletrodo para eletrofloculação são alumínio e ferro, de vez que são de baixo custo, facilmente disponíveis e de grande eficácia.

Foi conseguido um pequeno volume de amostra de efluente de água de produção do Centro de Pesquisas da Petrobras (CENPES), sendo que esta amostra foi utilizada para se determinar qual o melhor material do eletrodo para a realização dos testes com este efluente. Assim, foram testados eletrodos de ferro e de alumínio e determinada a eficiência de remoção dos seguintes parâmetros: O\&G, cor e turbidez.

A Figura 5 e 6 compara a eficiência do tratamento para estes dois tipos de eletrodos, mais de $98 \%$ dos poluentes foram retirados das águas residuais com uma concentração inicial de óleos e graxas de $190 \mathrm{mg} / \mathrm{L}$. O efluente com eletrodos de alumínio foi mais estável e mais clarificado e, enquanto o efluente com eletrodos de ferro apareceu primeiro com cor esverdeada e, logo após filtração, ficou amarelo e turvo. A cor verde é proveniente de Fe (II). O Fe (II) é o íon comum gerado in situ da eletrólise de ferro. Tem relativamente alta solubilidade em meio ácido ou $\mathrm{pH}$ neutro e pode ser oxidado facilmente em Fe (III) pelo oxigênio dissolvido na água.

Assim, o eletrodo de alumínio é melhor do que eletrodo de ferro para o presente trabalho. Por conseguinte, todos os experimentos subsequentes foram realizados com eletrodos de alumínio utilizando efluentes simulados.

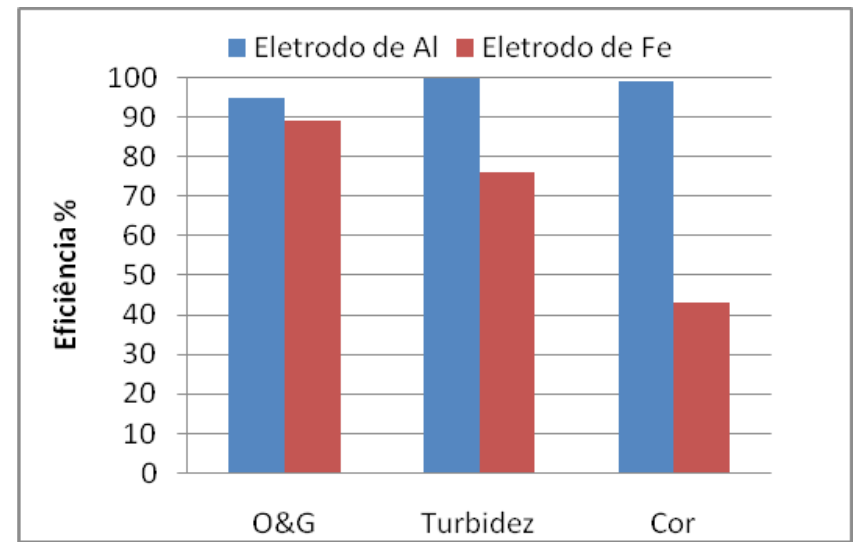

Figura 5- Comparação da eficiência do tratamento entre eletrodo de alumínio e eletrodo de ferro. Condições: efluente real de água de produção contendo: $O \& G=190$ (mg/L), turbidez $=810(\mathrm{NTU})$, cor $=1,442$ (Abs.), condutividade $=$ $123,4(\mathrm{mS} / \mathrm{cm})$ e $\mathrm{pH}=6,27$. Ensaios realizados utilizando eletrofloculação com corrente contínua com $5 \mathrm{~min}$. de eletrólise, intensidade de corrente $=5 \mathrm{~A}$ e distância entre eletrodos $=0,5 \mathrm{~cm}$.

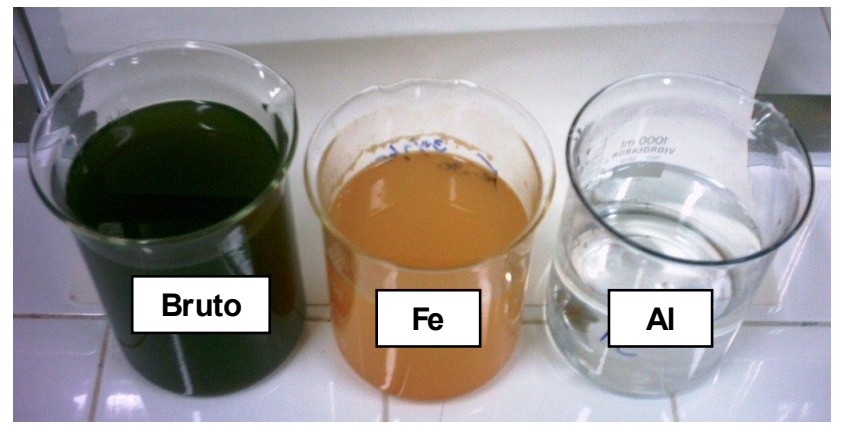

Figura 6: comparação entre eletrodo de alumínio e eletrodo de ferro após eletrólise de água de produção.

\subsection{Variação de tempo de eletrofloculação utilizando corrente alternada}

Os primeiros ensaios de EF com corrente alternada foram feitos utilizando $900 \mathrm{~mL}$ do efluente sintético em água salina contendo $1 \mathrm{~g}$ de óleo cru, $200 \mathrm{mg} / \mathrm{L}$ de emulsificante e $35 \mathrm{~g}$ de $\mathrm{NaCl}$ sem modificação de $\mathrm{pH}\left(\mathrm{pH}_{\text {inicial }}=6,5\right)$. A intensidade de corrente aplicada foi de $6 \mathrm{~A}$ e com variação do tempo de eletrólise de 5,10 e 15 minutos com o intuito de se observar a evolução do processo. Vide Tabela 2.

A eficiência de remoção de turbidez depende diretamente da concentração de íons 
produzidos pelos eletrodos. Quando se aumenta o tempo de operação, ocorre um aumento na concentração de íons e os seus flocos de hidróxido. Entretanto, foi observado que, após 5 minutos de eletrofloculação, já se obtém elevada remoção de turbidez não necessitando de tempos maiores de eletrólise.

Além disso, observa-se um aumento do $\mathrm{pH}$ durante o tratamento do efluente, o qual pode ser atribuído ao consumo de $\mathrm{H}^{+}$decorrente da formação de hidrogênio no catodo proveniente da eletrólise da água. Este resultado é esperado já que a eletrofloculação produz íons $\mathrm{OH}^{-}$.

O consumo teórico do eletrodo para os testes utilizando $6 \mathrm{~A}$ com 5,10 e 15 minutos de eletrólise foi de $0,18 \mathrm{~g} / \mathrm{L}, 0,37 \mathrm{~g} / \mathrm{L}$ e $0,56 \mathrm{~g} / \mathrm{L}$ respectivamente. A energia elétrica consumida foi de $0,0004 \mathrm{kWh} / \mathrm{L}, 0,0009 \mathrm{kWh} / \mathrm{L}$ e 0,0013 $\mathrm{kWh} / \mathrm{L}$ respectivamente. A frequência obtida do equipamento foi de $27 \mathrm{~Hz}$.

\subsection{Variação da intensidade de corrente}

Como os resultados anteriores foram satisfatórios, tentou-se otimizar a EF variando-se a intensidade de corrente: 5 e 10 A. Nestes ensaios, foram realizados testes com tempo de eletrólise de 5 minutos em $\mathrm{pH}_{\mathrm{i}} 6,5$. Os resultados apresentados na Tabela 5 demonstram que ocorreu uma ótima remoção de cor, turbidez e O\&G.

A Tabela 3 apresenta os resultados obtidos através da variação da intensidade de corrente com corrente alternada.

O aumento da intensidade de corrente aumenta a geração de hidrogênio e oxigênio formados na superfície do eletrodo. Isto leva para um aumento do número de bolhas de gás dentro da célula eletrolítica. Por conseguinte, a etapa de ligação entre as bolhas de gás e as gotas de óleo é aumentada. Entretanto, ainda com o aumento na corrente além do valor ideal, aumenta excessivamente o número de bolhas geradas.

De acordo com os resultados, fica evidente que, aumentando a intensidade de corrente de $5 \mathrm{~A}$ para $10 \mathrm{~A}$, a remoção de poluentes também aumenta. Ao aumentar à corrente, o fluxo de bolhas é aumentado, e por sua vez, a probabilidade de colisão é maior. Ao mesmo tempo, a concentração da dissolução do ânodo de íons metálicos também aumenta. Em valores elevados de corrente, o consumo de eletrodos é elevado. Assim, a corrente na faixa de 5A desempenha um papel importante na obtenção de resultados satisfatórios.

O consumo de massa de eletrodo e energia elétrica para os testes utilizando $5 \mathrm{~A}$ e $10 \mathrm{~A}$ com 5 minutos de eletrólise em CA foi de $0,15 \mathrm{~g} / \mathrm{L}$ e $0,31 \mathrm{~g} / \mathrm{L}$ respectivamente. A energia elétrica consumida foi de $0,0003 \mathrm{kWh} / \mathrm{L}$ e 0,0009 $\mathrm{kWh} / \mathrm{L}$ respectivamente. A frequência foi de $26 \mathrm{e}$ $31 \mathrm{~Hz}$ respectivamente.

Como foi observado, com intensidade de corrente com $5 \mathrm{~A}$ na $\mathrm{CA}$, já se tem alta eficiência de remoção de poluentes, não necessitando de intensidades de corrente maiores, o que significaria maior consumo de energia e maior desgaste do eletrodo.

\subsection{Ensaios preliminares utilizando corrente contínua}

Os resultados dos ensaios são mostrados na Tabela 4.

Em corrente contínua, a massa de eletrodo consumida de 5 e $10 \mathrm{~A}$ foi de $0,15 \mathrm{~g} / \mathrm{L}$ e $0,31 \mathrm{~g} / \mathrm{L}$ respectivamente e a energia elétrica consumida foi de $0,0006 \mathrm{kWh} / \mathrm{L}$ e $0,0015 \mathrm{kWh} / \mathrm{L}$ respectivamente.

Como foi observado, com intensidade de corrente de $5 \mathrm{~A}$ na $\mathrm{CC}$, já se obtém alta eficiência de remoção de poluentes, não necessitando de intensidades de corrente maiores. A Figura 7 demonstra a evolução do tratamento com CA e CC.

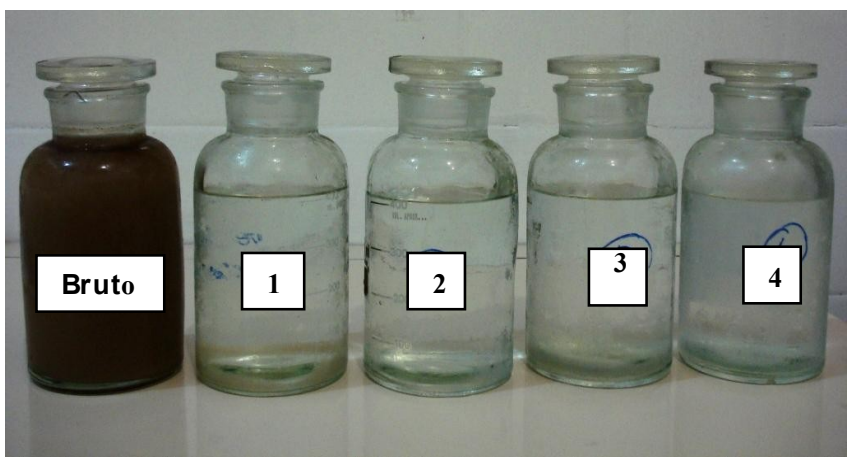

Figura 7 - Efluente bruto, 1) eletrofloculado com $\mathrm{CA}$ e $5 \mathrm{~A}$; 2) CA e 10A; 3) CC e 5A; 4) CC e 10A.

Com o objetivo de se aproximar do efluente real, foram feitos testes com efluente simulado com água do mar. Os ensaios foram feitos com CA e CC, o efluente sintético com água do mar $(900 \mathrm{~mL})$, contendo $0.9 \mathrm{~g}$ de óleo cru e $200 \mathrm{mg} / \mathrm{L}$ de emulsificante.

O ensaio foi realizado com modificação 
do $\mathrm{pH}_{\mathrm{i}}$ para 6,5, com a intenção de se reproduzir a condição do $\mathrm{pH}$ inicial dos ensaios anteriores. $\mathrm{O}$ abaixamento do $\mathrm{pH}$ foi realizado utilizando-se $\mathrm{H}_{2} \mathrm{SO}_{4} \quad 1 \mathrm{~N}$. Os resultados dos ensaios são mostrados na Tabela 5.

A massa de eletrodo consumida foi de $0,15 \mathrm{~g} / \mathrm{L}$ e o consumo de energia foi de 0,0003 $\mathrm{kWh} / \mathrm{L}$. A frequência obtida foi de $25 \mathrm{~Hz}$.

O resultado dos ensaios com corrente contínua com efluente simulado com água do mar são mostrados na Tabela 6.

A massa de eletrodo consumida em corrente contínua foi de $0,15 \mathrm{~g} / \mathrm{L}$ e o consumo de energia foi de $0,0006 \mathrm{kWh} / \mathrm{L}$.

A alta condutividade do meio favorece o processo de eletrolise, reduzindo a tensão aplicada aos eletrodos necessária para se obter correntes elevadas. O mesmo é confirmado nos ensaios realizados neste trabalho.

Segundo Otenio e colaboradores (2008), o pH e a condutividade são grandezas sempre associadas, pois a condutividade é diretamente proporcional à ionização de substancias dissolvidas no liquido. A ação da eletrólise tem também uma estreita relação com a condutividade e, consequentemente, com $\mathrm{opH}$, quanto maior a condutividade maior a transmissão de corrente.

Como se observa na Figura 8 , os resultados de eficiência de remoção de poluentes são bem parecidos, demonstrando a eficiência das duas tecnologias. Nas amostras 1 e 2, a eficiência de remoção foi elevada, mostrando uma pequena melhora de remoção em O\&G e uma maior clarificação para a amostra 1 .

Durante todas as eletrólises foi observada a deposição de uma camada de material esbranquiçado sobre os catodos, sendo que na água do mar o efeito foi aumentado. Esta camada foi menor no sistema de eletrólise de corrente alternada, possivelmente devido ao sistema realizar trocas sucessivas de polaridade por segundo, numa média de $28 \mathrm{~Hz}$, de acordo com os resultados anteriores. Esta camada também foi observada por Queiroz (1996), utilizando eletrodos de titânio em corrente contínua no tratamento da água de produção, sendo este depósito caracterizado como hidróxidos e carbonatos de sais presentes no meio.

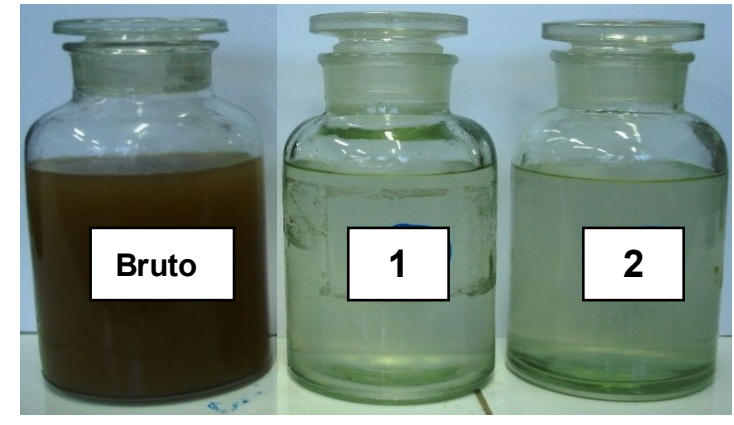

Figura 8: Efluente bruto, 1) $\mathrm{CA}$ e $\mathrm{pH}_{\mathrm{i}} 6,5$ e 2) $\mathrm{CC}$ e $\mathrm{pH}_{\mathrm{i}}$ 6,5. Tempo de eletrolise de $5 \mathrm{~min}$. e intensidade de corrente de $5 \mathrm{~A}$ para todos os ensaios.

$\mathrm{O} \mathrm{pH}$ da solução, que inicialmente foi de 6,5 , aumentou após os testes com água salina sintética até o fim da eletrólise, enquanto que nos ensaios com água do mar, o $\mathrm{pH}$ diminuiu. De acordo com Santos e colaboradores (2004), quando a camada de depósito no catodo se torna significativa tem-se a diminuição do $\mathrm{pH}$ da solução em função de que o material depositado diminui a liberação da hidroxila aumentando a concentração de ácido clorídrico na solução.

Queiroz (1996) propõe um conjunto de reações prováveis de acontecer a partir da eletrolise de efluente de alta salinidade. Equações (7 a 9).

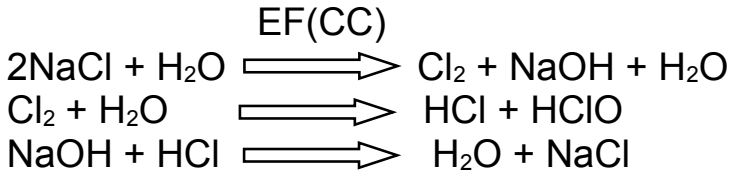

De acordo com Queiroz (1996), o ácido clorídrico formado, por sua vez, deveria ser totalmente neutralizado pelo hidróxido de sódio formado no momento da geração de $\mathrm{Cl}_{2}$, através da eletrólise. A presença de outros cátions, no entanto, pode neutralizar parte do hidróxido de sódio gerado, levando a alterações no pH. Este fato explica a constatação prática da queda do $\mathrm{pH}$ do meio contendo impurezas durante a eletrólise, ao contrario do que ocorre num processo de geração de cloro em uma solução de cloreto de sódio puro, quando o $\mathrm{pH}$ sobe, ocorrendo desprendimento de $\mathrm{H}_{2}$ acumulando-se $\mathrm{OH}^{-}$no meio.

O objetivo principal deste trabalho foi avaliar algumas condições experimentais que possam vir a ser empregadas na prática para 0 tratamento eletroquímico de compostos poluentes presentes em água de produção. 
Os resultados obtidos permitem visualizar a redução de $O \& G$, cor e turbidez para diferentes tempos de eletrólise e intensidade de corrente aplicados nos ensaios. Nas condições do experimento, a intensidade de corrente de $5 \mathrm{~A}$ e tempo de 5 minutos revelaram ser a mais indicada para remoção.

No presente trabalho, pôde-se confirmar que o processo de tratamento de eletrofloculação com corrente alternada através do efluente simulado de água de produção parece ser aplicável com resultados satisfatórios, tanto para lançamento em corpo receptor, como para a utilização da água tratada para reinjeção em poços de petróleo.

De uma forma geral, os resultados confirmam - potencial da técnica, onde através de equipamentos simples, compactos e de baixo custo operacional, possam ser empregados na descontaminação de compostos químicos.

Embora a análise completa ainda esteja em andamento, os resultados parciais desse trabalho apontam para a viabilidade técnica do processo. Objetiva-se trabalhar com variação de outros parâmetros do processo tais como: distância entre eletrodos, tempo, $\mathrm{pH}$, intensidade de corrente e variação de frequência.

\section{Referências}

1. BAYRAMOGLU, M.; KOBYA, M.; CAN, O. T.; SOZBIR, M., Operating cost analysis of electrocoagulation of textile dye wastewater, Separation and purification Technology, v. 37, p. 117-125, 2004.

2. CAMPOS, A. L. O.; RABELO, T. S.; SANTOS, R. O.; MELO, R. F. L. V., Produção mais limpa na indústria do petróleo: o caso da água produzida no campo de carmópolis. $23^{\circ}$ Congresso Brasileiro de Engenharia Sanitária e Ambiental (ABES), 2005.

3. CAN, O. T.; KOBYA, M.; DEMIRBAS, E.; BAYRAMOGLU, M., Treatment of the textile wastewater by combined electrocoagulation, Chemosphere, v. 62 , p. 181-187, 2006.

4. CHEN, X.; CHEN, G.; YUE, P. L., Separation of pollutants from restaurant wastewater electrocoagulation, Separation and
Purification Technology 19, p.65-76, 2000.

5. CONAMA, Conselho Nacional do Meio Ambiente. Resolução $\mathrm{n}^{\circ} 357 / 05$ e $\mathrm{n}^{\circ}$ 393/07.

6. CRESPILHO, F. N.; SANTANA, C. G.; REZENDE, M. O. O., Tratamento de efluente de indústria de processamento de coco utilizando eletroflotação. Química Nova, n. 27, v. 2, 2004.

7. FONSECA, M. R. M., Completamente Química, Físico-Química, Ed. FTD, São Paulo, 2001.

8. GABARDO, I. T., Caracterização química e toxicológica da água produzida descartada em plataformas de óleo e gás na costa brasileira e seu comportamento dispersivo no mar. Tese (Doutorado) - UFRN, 250f Natal, 2007.

9. GÜRSES, A.; YALÇIN, M.; DOğAR, C., Electrocoagulation of some reactive dyes: a statistical investigation of some electrochemical variables, Waste Management, Vol 22, n. 5, P. 491-499, 2002.

10. KIM, T. H.; PARK; C.; SHIN, E.B.; KIM, $S$., Decolorization of disperse and reactive dyes by continuous electrocoagulation process, Desalination, 150, p. 165-175, 2002.

11. KOBYA, M.; CAN, O. T.; BAYRAMOGLU, $M$., Treatment of textile wastewater by electrocoagulation usig iron and aluminum electrodes, Journal of Hazardous Materials B100, 163-178. 2003.

12. MARIANO, J. B., Impactos ambientais do refino de petróleo, Editora Interciência, Rio de janeiro, 2005.

13. MOLLAH, M.Y.A.; SCHENNACH, R.; PARGA, J. R.; COCKE, D. L., Electrocoagulation (EC): science and applications, Journal of Hazardous Materials, v. 84, p. 29-41, 2001.

14. MOLLAH, M. Y. A.; MORKOWSKY, P.; GOMES, J. A. G.; KESMEZ, M.; PARGA, J.; COCKE, D. L., Fundamentals, present and future perspectives of electrocoagulation, Journal of Hazardous Materials, B114, p. 199-210, 2004.

15. OTENIO, M. H.; PANCHONI, L. C.; CRUZ, G. C. A.; RAVANHANI, C.; 
BIDÓIA, E. D., Avaliação de escala laboratorial da utilização do processo eletrolítico no tratamento de águas. Química Nova, Vol. 31, n. 3, p. 508-513, 2008.

16. QUEIROZ, M. S.; SOUZA, A. D.; ABREU, E. S.; GOMES, N. T.; NETO, O. A. A., Aplicação do processo eletrolítico ao tratamento de água de produção, DITER/ SEBIO - CEMPES, n. 08, 1996.

17. RODRIGUES, J. R., Utilização de copolímeros a base de estireno e divinilbenzeno na extração de óleos e graxas presentes em efluentes aquosos. Dissertação de Mestrado, Instituto Militar de Engenharia, Rio de Janeiro, Brasil, 2007.

18. SANTOS, A. C.; SILVA, S. R., TONHOLO, J.; ZANTA, C. L. P. S., Tratamento de água produzida de petróleo através da tecnologia eletroquímica. $3^{0}$ Congresso Brasileiro de P\&D em Petróleo e Gás, Salvador, bahia, 2005.

19. SILVA, A. L. C. Processo eletrolítico: Uma alternativa para o tratamento de águas residuárias. Dissertação de Especialização em Química Ambiental, Universidade do Estado do Rio de Janeiro, Rio de Janeiro, RJ, Brasil. 2002.

20. VIEIRA, D. S.; CAMMAROTA, M. C.; CAMPORESE, E. F. S., Redução de contaminantes presentes na água de produção de petróleo, $2^{0}$ Congresso Brasileira de P\&D em petróleo e gás. Rio de janeiro, 2003.

21. VLYSSIDES, A. G.; KARLIS, P. K.; ZORPAS, A. A., Electrochemical oxidation of noncyanide strippers wastes, Environment International, Vol. 25, n. 5, P. 663-670, 1999.

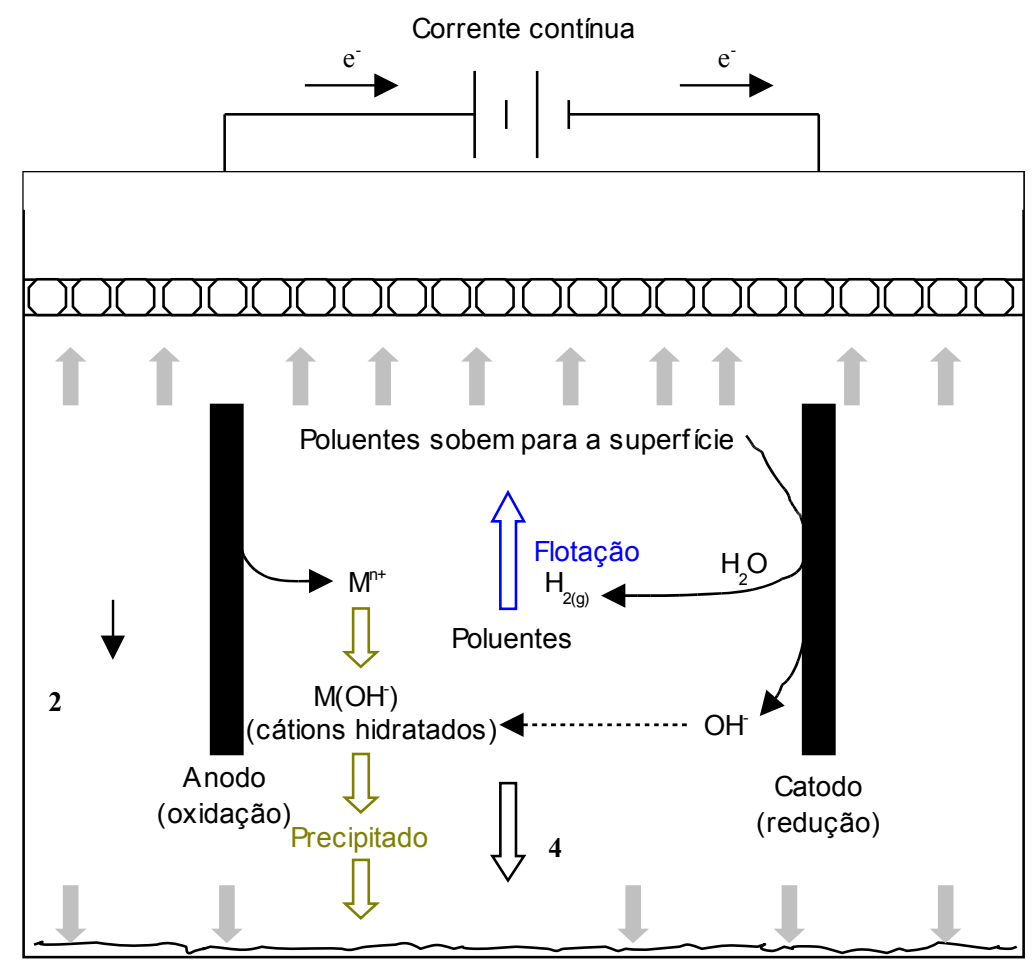

Figura 1 - Diagrama esquemático de célula de eletrofloculação com dois eletrodos (Mollah et al., 2004 


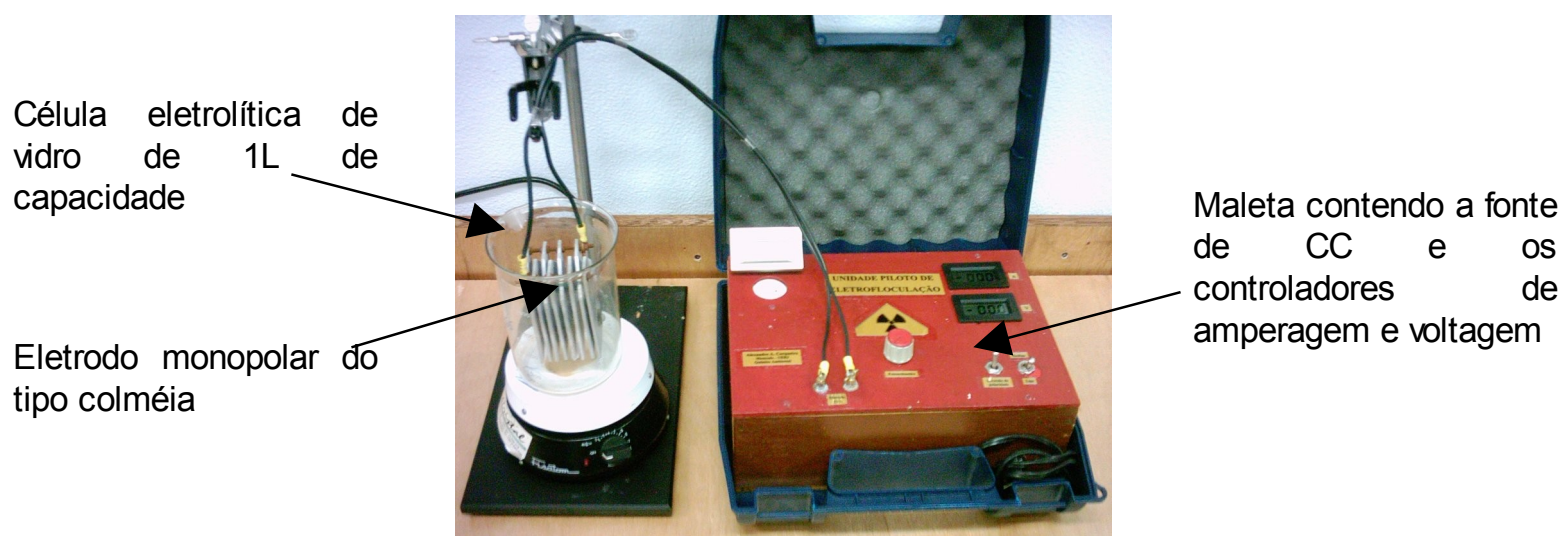

Figura 2: Unidade piloto de eletrofloculação com corrente contínua

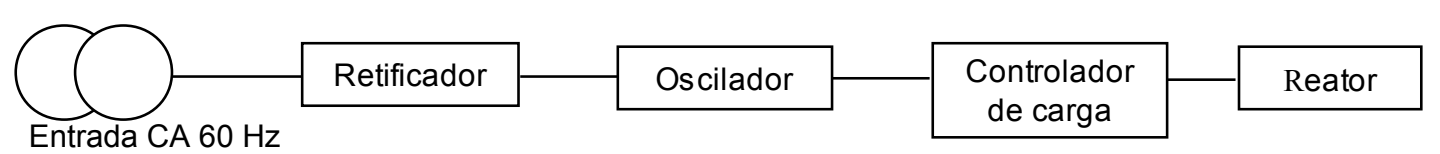

Figura 3 - Diagrama esquemático experimental de CA

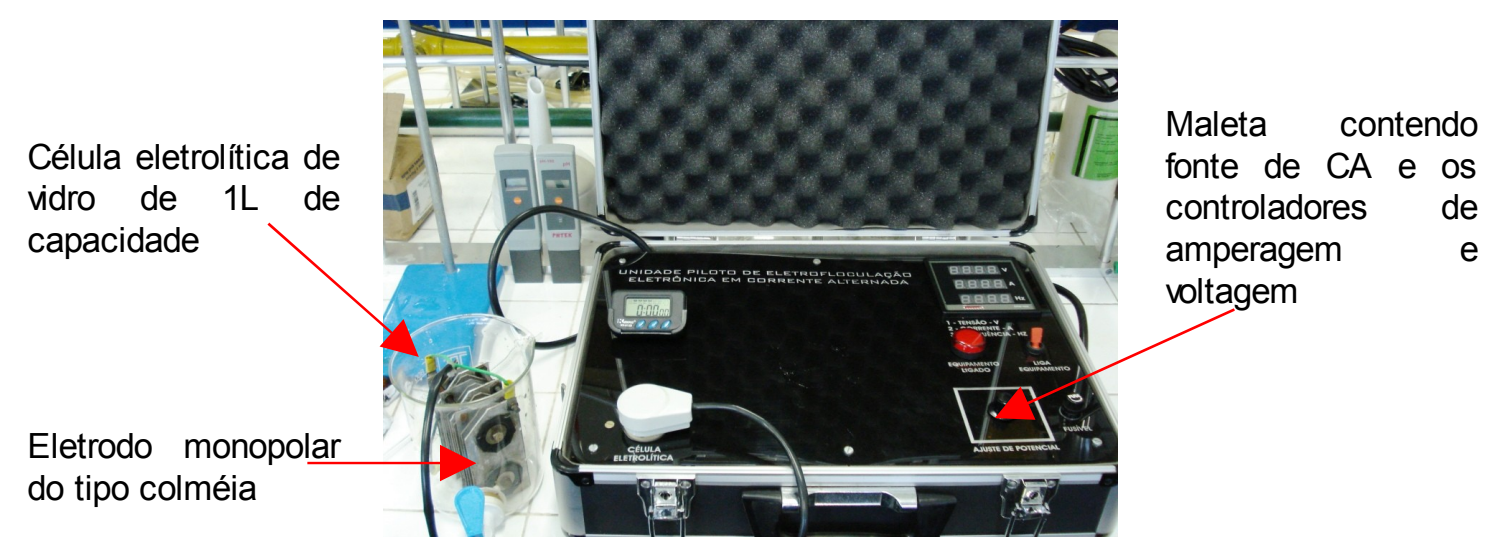

Figura 4 - Unidade piloto de eletrofloculação com corrente alternada 
Tabela 1 - Parâmetros obtidos durante a realização do efluente real e sintético em diferentes meios aquosos

\begin{tabular}{c|c|c|c}
\hline Parâmetro & Água de produção & Água salina $^{\text {a }}$ & Água do mar \\
\hline Cor (Absorbância) & 1,44 & 4,9 & 6,9 \\
\hline Turbidez (NTU) & 810 & 4900 & 5090 \\
\hline $\mathrm{pH}$ & 6,27 & 6,5 & 7,9 \\
\hline Condutividade $(\mathrm{mS} / \mathrm{cm})$ & 123,4 & 72,70 & 47,70 \\
\hline O\&G $(\mathrm{mg} / \mathrm{L})$ & 190 & 690 & 690 \\
\hline
\end{tabular}

a: água salina: $35.000 \mathrm{ppm}$ de $\mathrm{NaCl}$

Tabela 2 - Resultados obtidos após variação do tempo de EF com corrente alternada

\begin{tabular}{c|c|c|c|c}
\hline \multirow{2}{*}{ Parâmetro } & \multirow{2}{*}{ Efluente simulado } & \multicolumn{3}{|c}{ Eletrofloculado } \\
\cline { 3 - 5 } & & $5 \mathrm{~min}$ & $10 \mathrm{~min}$ & $15 \mathrm{~min}$ \\
\hline Turbidez & 4900 & 9,7 & 8,5 & 8,77 \\
\hline $\mathrm{pH}$ & 6,5 & 8,0 & 8,5 & 8,8 \\
\hline
\end{tabular}

condições: concentração salina: $35.000 \mathrm{mg} / \mathrm{L}$ de NaCl; i $=6 \mathrm{~A}$.

Tabela 3 - Resultados obtidos variando-se a intensidade de corrente utilizando corrente alternada.

\begin{tabular}{c|c|c|c}
\hline \multirow{2}{*}{ Parâmetro } & \multirow{2}{*}{ Efluente simulado } & \multicolumn{2}{|c}{ Eletrofloculado } \\
\cline { 3 - 4 } & & $5 \mathrm{~A}$ & $10 \mathrm{~A}$ \\
\hline O\&G (mg/L) & 690 & 20 & 10 \\
\hline Cor (Abs.) & 4,9 & 0,038 & 0,031 \\
\hline Turbidez (NTU) & 4900 & 16,2 & 8,6 \\
\hline $\mathrm{pH}$ & 6,5 & 8,1 & 8,5 \\
\hline Condutividade (mS/cm) & 72,7 & 68,15 & 68,85 \\
\hline
\end{tabular}

Condições: concentração salina: $35.000 \mathrm{mg} / \mathrm{L}$ de $\mathrm{NaCl}$ e tempo $5 \mathrm{~min}$.

Tabela 4 - Resultados obtidos variando-se a intensidade de corrente utilizando corrente contínua

\begin{tabular}{c|c|c|c}
\hline \multirow{2}{*}{ Parâmetro } & \multirow{2}{*}{ Efluente simulado } & \multicolumn{2}{|c}{ Eletrofloculado } \\
\cline { 3 - 4 } & 690 & $5 \mathrm{~A}$ & $10 \mathrm{~A}$ \\
\hline O\&G (mg/L) & 4,9 & 15 & 10 \\
\hline Cor (abs) & 4900 & 11,4 & 8,041 \\
\hline Turbidez (NTU) & 6,5 & 7,9 & 8,031 \\
\hline $\mathrm{pH}$ & 72,7 & 68,80 & 70,25 \\
\hline Condutividade $(\mathrm{mS} / \mathrm{cm})$ & &
\end{tabular}

Condições: concentração salina: $35.000 \mathrm{mg} / \mathrm{L}$ de $\mathrm{NaCl}$ e tempo $5 \mathrm{~min}$. 
Tabela 5 - Ensaios realizados em corrente alternada com efluente simulado com água do mar.

\begin{tabular}{c|c|c}
\hline Parâmetro & Efluente simulado & $\begin{array}{c}\text { Eletrofloculado com } \\
\text { corrente alternada }\end{array}$ \\
\hline Turbidez (NTU) & 5090 & 14,3 \\
\hline Cor (abs) & 6,94 & 0,069 \\
\hline O\&G (mg/L) & 690 & 29 \\
\hline $\mathrm{pH}$ & 7,9 & 4,6 \\
\hline Condutividade $(\mathrm{mS} / \mathrm{cm})$ & 47,4 & 50,9 \\
\hline
\end{tabular}

Condições: $\mathrm{i}=5 \mathrm{~A}$ e tempo de 5 min.

Tabela 6: Ensaios realizados em corrente contínua com água do mar.

\begin{tabular}{c|c|c}
\hline Parâmetro & Efluente simulado & $\begin{array}{c}\text { Eletrofloculado com } \\
\text { corrente contínua }\end{array}$ \\
\hline Turbidez (NTU) & 5090 & 35 \\
\hline Cor (Abs) & 6,94 & 0,129 \\
\hline O\&G (mg/L) & 690 & 40 \\
\hline $\mathrm{pH}$ & 7,9 & 4,7 \\
\hline Condutividade $(\mathrm{mS} / \mathrm{cm})$ & 47,4 & 51,7 \\
\hline
\end{tabular}

Condições: $\mathrm{i}=5 \mathrm{~A}$ e $\mathrm{t}=5 \mathrm{~min}$. 